\title{
COVID-19 Is an Independent Risk Factor for Acute Ischemic Stroke
}

\author{
(D) P. Belani, (D). Schefflein, (DS. Kihira, (D)B. Rigney, (D)B.N. Delman, (D). Mahmoudi, (D). Mocco, (D). Majidi, (D). Yeckley,
} (iD) A. Aggarwal, (DD. Lefton, and (D) A.H. Doshi

\begin{abstract}
BACKGROUND AND PURPOSE: Coronavirus disease 2019 (COVID-19) is an active worldwide pandemic with diverse complications. Stroke as a presentation has not been strongly associated with COVID-19. The authors aimed to retrospectively review a link between COVID-19 and acute stroke.
\end{abstract}

MATERIALS AND METHODS: We conducted a retrospective case-control study of 41 cases and 82 control subjects matched by age, sex, and risk factors. Cases were patients who underwent stroke alert imaging with confirmed acute stroke on imaging between March 16 and April 5, 2020, at 6 hospitals across New York City. Control subjects were those who underwent stroke alertimaging during the same timeframe without imaging evidence of acute infarction. Data pertaining to diagnosis of COVID-19 infection, patient demographics, and risk factors were collected. A univariate analysis was performed to assess the covariate effect of risk factors and COVID-19 status on stroke imaging with positive findings.

RESULTS: The mean age for cases and controls was $65.5 \pm 15.3$ years and $68.8 \pm 13.2$ years, respectively. Of patients with acute ischemic stroke, $46.3 \%$ had COVID-19 infection compared with $18.3 \%$ of controls $(P=.001)$. After adjusting for age, sex, and risk factors, COVID-19 infection had a significant independent association with acute ischemic stroke compared with control subjects (OR, 3.9; $95 \% \mathrm{Cl}$, 1.7-8.9; $P=.001)$.

CONCLUSIONS: We demonstrated that COVID-19 infection is significantly associated with imaging confirmation of acute ischemic stroke, and patients with COVID-19 should undergo more aggressive monitoring for stroke.

ABBREVIATIONS: COVID-19 = coronavirus disease 2019; SARS-CoV-2 = Severe Acute Respiratory Syndrome coronavirus 2; RT-PCR = reverse transcriptase polymerase chain reaction

I:

December 2019, a novel Severe Acute Respiratory Syndrome coronavirus 2 (SARS-CoV-2) emerged in Wuhan, China. ${ }^{1}$ Coronavirus disease 2019 (COVID-19), the clinical manifestation of SARS-CoV-2 infection, has since spread into a worldwide pandemic. ${ }^{2}$ According to the World Health Organization, as of April 22, 2020, at least 2.5 million confirmed cases of COVID-19 have been detected in 213 countries, with at least 169,000 patients dying from their disease or complications thereof. ${ }^{3}$

From the initial outbreak in China, transmission was identified from respiratory droplets and fomites, with symptoms initially

\footnotetext{
Received April 23, 2020; accepted after revision May 17.

From the Departments of Diagnostic, Molecular and Interventional Radiology (P.B., J.S., S.K., B.R., B.N.D., K.M., J.Y., A.A., D.L., A.H.D.), and Neurosurgery (J.M., S.M.), Icahn School of Medicine at Mount Sinai, New York, New York.

Please address correspondence to Puneet Belani, MD, Department of Diagnostic, Molecular and Interventional Radiology, Icahn School of Medicine at Mount Sinai and The Mount Sinai Hospital, One Gustave L. Levy Place, Box 1234, New York, NY 10029; e-mail: puneet.belani@mountsinai.org

- Indicates open access to non-subscribers at www.ajnr.org

http://dx.doi.org/10.3174/ajnr.A6650
}

reported as fever, dyspnea, cough, and severe hypoxia. With greater clinical experience, a broader spectrum of symptoms has emerged, such as gastrointestinal disease, headache, altered mental status, anosmia, and confusion. ${ }^{4-6}$ An acute hemorrhagic necrotizing encephalopathy has also been noted secondary to COVID-19 infection. ${ }^{7}$

Even before COVID-19, antecedent respiratory infections have been known to increase the short-term risk of ischemic stroke. ${ }^{8-16}$ Early reports from China indicate that neurologic symptoms are seen in approximately $36 \%$ of patients hospitalized with COVID-19. ${ }^{16}$ Poor outcomes with COVID-19 infection have an association with vascular risk factors such as hypertension, coronary artery disease, and diabetes mellitus. ${ }^{17}$ It has been proposed that the SARS-CoV-2 virus can cause a cytokine storm through angiotensin-converting enzyme 2 receptor binding, leading to a hypercoagulable state and an increased incidence of vascular thromboses in patients with COVID-19. ${ }^{18,19}$

To our knowledge, no peer-reviewed study has been published in the literature evaluating the incidence of acute ischemic stroke associated with COVID-19 when controlling for traditional 
vascular risk factors. With the dramatic rise in patients with COVID-19 in New York City in March 2020, the authors began to observe a disproportionately greater incidence of stroke among patients with COVID-19 compared with the non-COVID-19 population. The authors believe there may be an association between COVID-19 infection and stroke risk. In this study, our objectives were the following: 1) to determine whether infection with COVID-19 is indeed associated with increased incidence of stroke, and 2) to assess whether COVID-19 is an independent risk factor for stroke when controlling for conventional vascular risk factors.

\section{MATERIALS AND METHODS \\ Patient Selection}

The project was conducted as a retrospective case-control study with data gathered from 6 hospitals spread across the boroughs of Manhattan, Queens, and Brooklyn in New York City. The 6 hospitals consisted of 1 large academic hospital and 5 smaller community hospitals. Institutional review board approval was obtained on an expedited basis. A total of 139 patients had stroke alert for acute neurologic symptoms between March 16 and April 5 , 2020. All patients with stroke alert had a noncontrast head CT performed followed by vascular imaging (generally a CT angiography) unless there was a medical contraindication or clinical instability. Patients were included for the following reasons: 1) They had activated stroke alert, 2) CT head and vascular imaging, and 3) clinical data documenting COVID-19 status. Patients presenting primarily with hemorrhage, tumor, and vasculitis were excluded $(n=12)$. Patients with no clinical data were excluded $(n=1)$. This step yielded a total of 126 patients. Patients with acute ischemic stroke (small- or large-vessel occlusions) were matched with patients without stroke by age, sex, and major vascular risk factors on a 1:2 case-to-control ratio, which resulted in 41 cases and 82 control patients ( 3 patients were excluded from the control group to maintain this ratio). Thus, the final cohort number was 123 patients. The presence or absence of stroke was confirmed on CT and/or MR imaging by a board-certified radiologist with fellowship training in neuroradiology. Patients were deemed to have acute stroke on imaging if they had acute loss of gray-white differentiation on CT or a diffusion-restricting focus on MR imaging consistent with infarct. If the initial CT findings were negative but there was a persistent clinical suspicion for stroke, it was confirmed or refuted on brain MR imaging unless there was a contraindication.

Control subjects were defined as inpatients and those in the emergency department with negative neurologic findings on imaging (CT or MR imaging) for acute stroke in the setting of a clinical suspicion for acute stroke. Patients with initial negative imaging findings were followed up clinically and also on repeat imaging (if deemed clinically necessary) to verify the absence of acute stroke. Vascular risk factors, which were obtained from the medical record, included hypertension, coronary artery disease, diabetes mellitus type 2 , atrial fibrillation, congestive heart failure, dyslipidemia, current or former smoking status, evidence of prior stroke, and body mass index corresponding to overweight $\left(25-29.9 \mathrm{~kg} / \mathrm{m}^{2}\right)$ or obesity $\left(\geq 30 \mathrm{~kg} / \mathrm{m}^{2}\right)$. Data pertaining to a concurrent diagnosis of COVID-19 infection and patient demographics were also collected from the electronic medical record. COVID-19 infection was confirmed by in-house in vitro reverse transcriptase polymerase chain reaction (RT-PCR) of nasopharyngeal swabs performed on the cobas $6800 / 8800$ systems (Roche Diagnostics) under emergency authorization from the US Food and Drug Administration. Patients were considered negative for COVID-19 negative if the RT-PCR test findings were negative.

\section{Statistical Analysis}

Patients whose imaging and clinical findings were consistent with acute ischemic infarct were matched by age, sex, and the number of major vascular risk factors against controls whose imaging and clinical findings were inconsistent with acute ischemic infarct. There were 2 controls for every case.

A Pearson $\chi^{2}$ test was performed for categoric variables, and a $t$ test was performed for continuous variables for evaluation of the statistical difference between case and control groups. All tests were 2 -tailed, and a $P$ value of .05 was considered statistically significant. All statistical analyses were conducted with the SPSS statistical package for Windows, Version 25 (IBM).

\section{RESULTS}

\section{Clinical Characteristics of the Patient Population}

Stroke alerts were most commonly activated for patients with hemiplegia, hemisensory loss, dysarthria, facial droop, aphasia, and acute altered mental status. Our patient population consisted of 123 patients, of whom 41 patients who had an acute ischemic stroke (anterior or posterior circulation) were matched for age, sex, and vascular risk factors with 82 patients without stroke. The mean age for cases and controls was $65.5 \pm 15.3$ years and $68.8 \pm$ 13.2 years, respectively. Sex composition was identical between groups, with $56.1 \%$ men in each.

\section{Risk Factor Analysis}

There was no statistically significant difference in age, sex, or major vascular risk factors between case and control subject groups (Table). COVID-19 infection was confirmed in 19 of 41 patients (46.3\%) with acute ischemic stroke, compared with 15 of 82 patients (18.3\%) in the control group. The COVID-19 infection rate was significantly higher in patients with acute ischemic stroke compared with control subjects $(P=.001)$.

After we adjusted for age, sex, and major vascular risk factors, COVID-19 infection was found to be independently and significantly associated with patients with acute ischemic stroke compared with control subjects with an OR of 3.9 (95\% CI, 1.7-8.9; $P=.001)$.

\section{DISCUSSION}

We found that COVID-19 is an independent risk factor for imaging-confirmed acute ischemic stroke during stroke alerts evaluation, after controlling for traditional vascular risk factors. This finding suggests that COVID-19 infection is associated with increased morbidity and mortality that transcends the primary cardiopulmonary sequelae from the infection. It has been observed that a large number of patients affected with COVID-19 have underlying vascular disease. A meta-analysis of 6 published 
Demographic characteristics of case and control subjects

\begin{tabular}{lllc}
\hline \multicolumn{1}{c}{ Variables } & Cases $(\boldsymbol{n}=\mathbf{4 1})$ & \multicolumn{1}{c}{ Controls $(\boldsymbol{n}=\mathbf{8 2})$} & $\boldsymbol{P}$ Value \\
\hline Age (mean) (yr) & \multicolumn{1}{c}{$65.6 \pm 15$} & \multicolumn{1}{c}{$68.8 \pm 13$} & .24 \\
Male sex & $56.1 \%(23)$ & $56.1 \%(46)$ & 1.00 \\
COVID-19 (+) & $46.3 \%(19)$ & $18.3 \%(15)$ & .001 \\
Diabetes mellitus type 2 & $48.8 \%(20)$ & $43.9 \%(36)$ & .61 \\
Hypertension & $73.2 \%(30)$ & $73.2 \%(60)$ & 1.00 \\
Coronary artery disease & $29.3 \%(12)$ & $34.1 \%(28)$ & .59 \\
Congestive heart failure & $12.2 \%(5)$ & $15.9 \%(13)$ & .59 \\
Dyslipidemia & $43.9 \%(18)$ & $46.3 \%(38)$ & .80 \\
Atrial fibrillation & $29.3 \%(12)$ & $18.3 \%(15)$ & .18 \\
Prior stroke & $22 \%(9)$ & $31.7 \%(26)$ & .26 \\
BMI = 25-29.9 kg/m ${ }^{2}$ & $22 \%(9)$ & $32.9 \%(27)$ & .21 \\
BMI $>30 \mathrm{~kg} / \mathrm{m}^{2}$ & $39 \%(16)$ & $23.2 \%(19)$ & .09 \\
Smoking status & $34.1 \%(14)$ & $28.0 \%(23)$ & .31 \\
Current & $12.2 \%(5)$ & $7.3 \%(6)$ & .29 \\
Former & $22 \%(9)$ & $20.7 \%(17)$ & .53 \\
\hline
\end{tabular}

Note:-BMI indicates body mass index.

studies from China including 1527 patients with COVID-19 reported common comorbid conditions, including diabetes (9.7\%), cardiovascular disease (16.4\%), and hypertension (17.1\%). ${ }^{20}$ In particular, patients with severe COVID-19 had a 3fold higher incidence of cardiovascular disease than those with mild-to-moderate disease.

Multiple studies have reported that recent respiratory infections are known to increase the short-term risk of ischemic stroke. ${ }^{8,9,11,12,14,15}$ For example, Grau et al ${ }^{11}$ reported that bacterial and viral infections were risk factors for embolic and thrombotic infarcts. They found that infection within the preceding week was a risk factor for cerebral ischemia in age-adjusted multiple logistic regression analysis (OR, 2.9; 95\% CI, 1.31-6.4). Of 221 patients with COVID-19 at a hospital in Wuhan, ${ }^{20} 11(5 \%)$ developed acute ischemic stroke. This patient group was significantly older and more likely to have cardiovascular risk factors and severe COVID-19 infection. They were more likely to have an increased inflammatory response and a hypercoagulable state manifested by elevated C-reactive protein and D-dimer levels. ${ }^{21}$

Our study attempts to evaluate the impact of COVID-19 on stroke irrespective of other vascular risk factors. Patients with acute stroke and controls without stroke belonged to the same medical system and clinical setting. Our study confirms that there was a significantly greater incidence of acute ischemic stroke in patients with COVID-19 infection compared with those without the infection. After we adjusted for major vascular risk factors, a diagnosis of COVID-19 was associated with significantly more cases than in control subjects.

Several theories link infectious/inflammatory syndromes with an increased risk of stroke, probably due to the different mechanisms involving prothrombotic state, changes in lipid metabolism and platelet aggregation, alterations in endothelial function, and plaque instability and rupture. ${ }^{22,23}$ The current favored mechanism involves SARS-CoA-2 binding to the angiotensin-converting enzyme 2 receptor, potentially leading to a cytokine storm and ultimately to a hypercoagulable state in patients with COVID-19. ${ }^{18,19}$ Additionally, critically ill patients with SARSCoV-2 often show elevated D-dimer levels and platelet counts, which may render patients prone to acute cerebrovascular disease. $^{24}$ It has been shown in a mouse influenza model that after the selective blocking of cytokines, there was a reduction in infarction volume and improved survival. ${ }^{25}$

Limitations of our study are those that apply to all retrospective case-control studies. We tried to limit confounding variables like demographics and medical risk factors for stroke to reduce bias. We reduced referral bias by incorporating stroke alert cases from multiple hospitals across various communities in New York City. Another limitation is our sample size of 123 patients; however, these are all patients with stroke alerts presenting at 6 hospitals, which consisted of 1 large academic hospital and 5 smaller community hospitals, during a short time span of 2.5 weeks. Additionally, it is possible that some patients with clinical and radiologic signs of infection who tested positive for COVID-19 on RT-PCR could be falsely positive and with other viral or bacterial respiratory infections instead.

The sensitivity and specificity of the RT-PCR test used for the diagnosis of COVID-19 has not been definitively established because the test is approved under emergency use authorization. It is also possible that we may have under-represented the number of patients with COVID-19 (ie, clinically asymptomatic carriers who were not tested for COVID-19), but this could affect both the stroke and nonstroke outcomes. Finally, another important consideration is the possibility of unexpected selection bias with higher thresholds for presentation to the hospital due to social distancing/fear of a hospital setting during the pandemic. This coupled with an overall increased prevalence of COVID-19 may confound the true effect of COVID-19 on stroke risk.

\section{CONCLUSIONS}

To our knowledge, this is the first study to link SARS-CoV-2 with an increased risk of imaging-confirmed acute ischemic stroke when accounting for confounding risk factors. Future endeavors may assess whether this relationship holds true in a larger population and with the pathophysiologic mechanisms (such as the proinflammatory prothrombotic state and cytokine storm) inherent in COVID-19 that drive this association. Attempts should also be made to see whether the association holds true for largevessel and small-vessel strokes. Patients with COVID-19 should be evaluated early for acute neurologic changes, and timely workup should be performed in patients suspected of having stroke to reduce morbidity and mortality.

Disclosures: Bradley N. Delman-UNRELATED: Payment for Lectures Including Service on Speakers Bureaus: Bayer HealthCare Pharmaceuticals, Comments: ad hoc lectures, averaging 1 per year. Daniel Lefton-UNRELATED: Expert Testimony: Various.

\section{REFERENCES}

1. Huang C, Wang Y, Li X, et al. Clinical features of patients infected with 2019 novel coronavirus in Wuhan, China. Lancet 2020;395:497-506 CrossRef Medline

2. World Health Organization. WHO Director-General's opening remarks at the media briefing on COVID-19 20 May 2020. May 20, 
2020. https://www.who.int/dg/speeches/detail/who-director-generals-opening-remarks-at-the-media-briefing-on-covid-19-20-may2020. Accessed March 11, 2020

3. World Health Organization. Coronavirus disease 2019. 2020 April 30. https://www.who.int/emergencies/diseases/novel-coronavirus2019. Accessed April 30, 2020

4. Mahajan A, Hirsch JA. Novel coronavirus: what neuroradiologists as citizens of the world need to know. AJNR Am J Neuroradiol 2020;41:552-54 CrossRef Medline

5. Jiang F, Deng L, Zhang L, et al. Review of the clinical characteristics of coronavirus disease. J Gen Intern Med 2020;35:1545-49 CrossRef Medline

6. World Health Organization. COVID-19: Operational guidance for maintaining essential health services during an outbreak. March 25, 2020. https://www.who.int/publications-detail/covid-19-operationalguidance-for-maintaining-essential-health-services-during-anoutbreak. Accessed March 25, 2020

7. Poyiadji N, Shahin G, Noujaim D, et al. COVID-19-associated acute hemorrhagic necrotizing encephalopathy: CT and MRI features. Radiology 2020 Mar 31. [Epub ahead of print] CrossRef Medline

8. Zurrú MC, Alonzo C, Brescacín L, et al. Recent respiratory infection predicts atherothrombotic stroke: case-control study in a Buenos Aires healthcare system. Stroke 2009;40:1986-90 CrossRef Medline

9. Cowan LT, Lutsey PL, Pankow JS, et al. Inpatient and outpatient infection as a trigger of cardiovascular disease: the ARIC study. $J$ Am Heart Assoc 2018;7:e009683 CrossRef Medline

10. Grau AJ, Buggle F, Heindl S, et al. Recent infection as a risk factor for cerebrovascular ischemia. Stroke 1995;26:373-79 CrossRef Medline

11. Grau AJ, Buggle F, Becher $\mathrm{H}$, et al. Recent bacterial and viral infection is a risk factor for cerebrovascular ischemia: clinical and biochemical studies. Neurology 1998;50:196-203 CrossRef Medline

12. Bova IY, Bornstein NM, Korczyn AD. Acute infection as a risk factor for ischemic stroke. Stroke 1996;27:2204-06 CrossRef Medline

13. Clayton TC, Thompson M, Meade TW. Recent respiratory infection and risk of cardiovascular disease: case-control study through a general practice database. Eur Heart $J$ 2008;29:96-103 CrossRef Medline
14. Lindsberg PJ, Grau AJ. Inflammation and infections as risk factors for ischemic stroke. Stroke 2003;34:2518-32 CrossRef

15. Becher $\mathrm{H}$, Grau A, Steindorf K, et al. Previous infection and other risk factors for acute cerebrovascular ischaemia: attributable risks and the characterisation of high-risk groups. J Epidemiol Biostat 2000;5:277-83 Medline

16. Mao L, Jin H, Wang $M$, et al. Neurologic Manifestations of Hospitalized Patients with Coronavirus Disease 2019 in Wuhan, China. JAMA Neurol 2020 April 10. [Epub ahead of print] CrossRef Medline

17. Zhou F, Yu T, Du R, et al. Clinical course and risk factors for mortality of adult inpatients with COVID-19 in Wuhan, China: a retrospective cohort study. Lancet 2020;395:1054-62 CrossRef

18. Mehta P, McAuley DF, Brown M, et al. COVID-19: consider cytokine storm syndromes and immunosuppression. Lancet 2020;395: 1033-4 CrossRef Medline

19. Connors JM, Levy JH. COVID-19 and its implications for thrombosis and anticoagulation. Blood 2020 Apr 27. [Epub ahead of print] CrossRef

20. Li B, Yang J, Zhao F, et al. Prevalence and impact of cardiovascular metabolic diseases on COVID-19 in China. Clin Res Cardiol 2020; 109:531-38 CrossRef Medline

21. Avula A, Nalleballe K, Narula N, et al. COVID-19 presenting as stroke. Brain Behav Immun 2020 Apr 28. [Epub ahead of print] CrossRef Medline

22. Ameriso SF, Wong VL, Quismorio FP, et al. Immunohematologic characteristics of infection-associated cerebral infarction. Stroke 1991;22:1004-09 CrossRef Medline

23. Mittleman MA, Mostofsky E. Physical, psychological and chemical triggers of acute cardiovascular events: preventive strategies. Circulation 2011;124:346-54 CrossRef Medline

24. Wu Y, Xu X, Chen Z, et al. Nervous system involvement after infection with COVID-19 and other coronaviruses. Brain Behav Immun 2020 Mar 30. [Epub ahead of print] CrossRef Medline

25. Muhammad S, Haasbach E, Kotchourko $M$, et al. Influenza virus infection aggravates stroke outcome. Stroke 2011;42:783-91 CrossRef Medline 